\title{
Childhood trauma and chronic illness in adulthood: mental health and socioeconomic status as explanatory factors and buffers
}

\author{
Steven E. Mock* and Susan M. Arai \\ Department of Recreation and Leisure Studies, Department of Health Studies and Gerontology, University of Waterloo, Waterloo, ON, Canada
}

\section{Edited by:}

Elysia Davis, University of California at Irvine, USA

\section{Reviewed by:}

Nadine Fink, Harvard Medical School, USA

Kerry-Ann Grant, Macquarie University, Australia

\section{*Correspondence:}

Steven E. Mock, Department of Recreation and Leisure Studies, Department of Health Studies and Gerontology, University of Waterloo, 200 University Avenue West, Waterloo, ON, Canada N2L 3G1.

e-mail:smock@uwaterloo.ca

\begin{abstract}
Experiences of traumatic events in childhood have been shown to have long-term consequences for health in adulthood. With data from the 2005 Canadian Community Health Survey we take a life course perspective of cumulative disadvantage and examine the potential role of mental health and socioeconomic status in adulthood as multiple mediators of the link between childhood trauma and chronic illness in adulthood. Mental health and socioeconomic status are also tested as buffers against the typically adverse consequences of childhood trauma. The results suggest mental health and socioeconomic status partially explain the association of childhood trauma with chronic illness in adulthood, with mental health showing a stronger effect. In addition, an analysis of the interactions suggested higher socioeconomic status is a potential protective factor for those with a history of trauma. Results also suggest cumulative disadvantage following trauma may lead to chronic illness and suggest the need for public health expenditures on resources such as counseling and income supports to prevent or reduce psychological harm and chronic illness resulting from traumatic events.
\end{abstract}

Keywords: childhood trauma, chronic illness, mental health, socioeconomic status, cumulative disadvantage

\section{INTRODUCTION}

The experience of traumatic events in childhood has consequences for health in adulthood. A broad range of traumatic events experienced in childhood including physical abuse, sexual abuse, prolonged hospitalization, and family instability such as parental unemployment or substance abuse have been linked to chronic illness in adulthood stemming from poor immune functioning or poor cardiovascular health (Springer et al., 2003; Mulvihill, 2005; O'Rand and Hamil-Luker, 2005; Wickrama et al., 2005). According to a life course perspective, there are multiple potential pathways that may contribute to a process of cumulative disadvantage following traumatic childhood events (Dannefer, 2003; Pearlin et al., 2005). One potential pathway is through the negative mental health consequences in adulthood of trauma experienced in childhood (Turner and Lloyd, 1995; Paolucci et al., 2001; Briere and Jordan, 2009). Recent work also suggests that there are socioeconomic disadvantages in adulthood resulting from trauma early in life that may contribute to chronic illness (Hyman, 2000; Zielinski, 2009; Currie and Spatz Widom, 2010). In addition to being potential explanatory factors for the link between childhood trauma and chronic illness, adult mental health and socioeconomic status (SES) are plausible buffers of the negative effects of trauma on health (Melchior et al., 2007; Wethington et al., 2008).

A traumatic experience is one that threatens death or injury to self or others and elicits intense feelings of fear, helplessness, or horror (American Psychiatric Association, 2000). Examples of traumatic events include physical abuse, sexual abuse, combat, witnessing violence or disaster, or the sudden death of a loved one. Over a lifetime most people experience at least one potentially traumatic event (Klein and Alexander, 2009). In a community-level study in the United States, it was found that $74.2 \%$ of women and $81.3 \%$ of men reported experiencing at least one traumatic event in their lifetime (Stein et al., 1997). A community study (Breslau, 2009; Breslau et al., 1999) reported similar findings, 78.1\% for women and $92.2 \%$ among men, for lifetime prevalence of exposure to traumatic events. Results from a national study in the United States indicate overall rates of child maltreatment at $13.8 \%$ (8.0\% having experienced sexual abuse, $6.7 \%$ physical abuse, $2.9 \%$ severe neglect, and $3.2 \%$ reporting multiple forms of child maltreatment experienced; Zielinski, 2009). Goodwin et al. (2003) reported physical abuse by parents at a rate of $15.8 \%$ and similar rates have been reported in Canadian research (MacMillan et al., 1997). In a community-level study of traumatic experiences in childhood (Turner and Lloyd, 1995) 19.5\% reported major illness or accident, $9.6 \%$ parental divorce, $17.7 \%$ repeating a year of school, $9.4 \%$ parent unemployment, $26.5 \%$ experience of a significant scare, $2.6 \%$ being sent away from home, $8.2 \%$ parent alcohol or drug use, and 3.8\% physical abuse.

The experience of traumatic events in childhood is associated with long-term health effects. In a study of the impact of childhood trauma on health in adulthood, those who reported childhood psychological or physical victimization had worse health than those who did not and also experienced significantly more decline in health over a 10-year period in adulthood (Greenfield and Marks, 2009). This is consistent with research that shows the negative impact of childhood adversity on cardiovascular health (O'Rand and Hamil-Luker, 2005) and recent reviews documenting a consistent association between childhood trauma and negative health consequences in adulthood including pain, unexplained somatic symptoms, stress-related illnesses, and illnesses related to 
suppressed immune functioning (Springer et al., 2003; Mulvihill, 2005). A life course perspective on cumulative disadvantage can help to explain the link between childhood trauma and chronic illness in adulthood. Specifically, a life course perspective takes into account individual, social, and historic factors that shape development (Elder, 1994) and consistent with this view, cumulative disadvantage is the notion that early disadvantage or negative experiences are linked to a cascade of adverse events throughout life that have long-term negative consequences (Dannefer, 2003; O'Rand and Hamil-Luker, 2005).

Two potential pathways that link experience of trauma in childhood with chronic illness in adulthood are mental health and SES. Child maltreatment increases the risk of behavior problems, including internalizing (anxiety, depression) and externalizing (aggression, acting out) behavior (Gilbert et al., 2009). Multiple studies have shown associations between childhood trauma and later psychological distress, psychiatric disorders, and depression (Turner and Lloyd, 1995; Pine and Cohen, 2002; Gilbert et al., 2009; Greenfield, 2010). Consequently, poor mental health has been shown to cause negative health outcomes in adulthood (Taylor, 2010). Pearlin et al. (2005) identified SES as a particularly important component of the process of cumulative disadvantage and poor health outcomes. A growing body of research suggests those with a history of childhood trauma face later economic and structural disadvantages in adulthood. Several authors note that lower income levels in adulthood for individuals who have experienced violence and maltreatment in childhood or adolescence is also the result of lower educational and occupational attainment (Hyman, 2000; MacMillan, 2000; Zielinski, 2009; Currie and Spatz Widom, 2010; Kunst et al., 2010). Individuals reporting more than one type of childhood maltreatment are more likely to be unemployed in adulthood or to have had a job loss in their family that resulted in financial hardship (Zielinski, 2009). Compared to individuals who have not experienced maltreatment in childhood, Zielinski (2009) found that adults who experienced physical abuse, mental abuse, or severe neglect in childhood were more than twice as likely to fall below the federal poverty level in adulthood and to live in a household with income in the lowest quartile of the distribution. Low SES among parents was also identified as a risk factor for the perpetration of maltreatment suggesting a potential mechanism in the intergenerational transmission of violence (Zielinski, 2009).

In sum, we set out to examine the association of childhood trauma with chronic illness in adulthood and identify potential mediators of any significant association found. Guided by the life course perspective and a model of cumulative disadvantage, we propose that mental health and SES in adulthood may be explanatory factors in the association between childhood trauma and chronic illness later in life. Finally, we test the potential role of mental health and SES as buffers against the typically adverse consequences of childhood trauma.

\section{MATERIALS AND METHODS DATA SOURCE}

Data were drawn from the public use version of the 2005 Canadian Community Health Survey (CCHS Cycle 3.1; Statistics Canada, 2006a). The primary aims of the CCHS are to collect information on health status, health care use, and determinants of health in the Canadian population. Data were collected in 2005 and include information on approximately 130,000 Canadians age 12 and older, excluding institutional residents, those who lived on reserves, and full-time members of the Canadian Forces.

\section{MEASURES}

\section{Traumatic events in childhood}

A sub-set of participants in the CCHS (participants in Manitoba and Saskatchewan) were asked about the occurrence of seven potentially traumatic events in childhood (Statistics Canada, 2006b) modeled after the measurement of lifetime traumas by Turner and Lloyd (1995). Specifically, participants were asked if they had experienced any of seven traumatic events as a child or a teenager before moving out of the house including: spent 2 or more weeks in the hospital, parental divorce, prolonged parental unemployment, experience of an event that scared the participant and led to thoughts about the event for years after, sent away from home for doing something wrong, family problems related to parental abuse of alcohol or drugs, and physically abused by someone close to the participant. Thus, the possible range of childhood traumas was $0-7$ and responses were summed to yield a total trauma score and then re-coded to yield a measure of no childhood trauma (0), one childhood trauma (1), and 2 or more traumatic events in childhood (2). In addition to the restriction of the sample size by the childhood stressors module, we also restricted the sample to working-age adults (age 18-65), yielding a sample size of 9,810.

\section{Demographic characteristics}

Age is grouped by categories which for the present analyses range from 1 (18-19 years) to 10 (60-64 years). Gender is denoted by female (1) and male (0). Visible minority status is coded as a contrast between white/European (0) and visible minority racial identity or ethnicity (i.e., Chinese, South Asian, Black, Filipino, Latin American, South East Asian, Arab, West Asian, Japanese, Korean, and other). Married/cohabiting indicates that the participant is married or cohabiting with a partner (1) compared to single, separated, divorced, or widowed (0). Not working refers to those who did not have a job in the past week (1) compared to those who are currently employed, on permanent disability, or retired (0).

\section{Mental health}

Current mental health was assessed with responses to the question, "In general, would you say your mental health is: excellent, very good, good, fair, or poor?" Responses were rated on a scale from 1 to 5 and coded such that higher values reflected greater levels of well-being.

\section{Socioeconomic status}

Education measures the highest level of education achieved including less than secondary school (1), secondary school graduation (2), some post-secondary (3), and post-secondary education (4). Income denotes an annual income of: less than $\$ 15,000(1), \$ 15,000$ to less than $\$ 30,000$ (2), $\$ 30,000$ to less than $\$ 50,000$ (3), $\$ 50,000$ to less than $\$ 80,000$ (4), or $\$ 80,000$ or greater (5). The education and income scores were transformed to $z$-scores and the mean of the $z$-scores was computed to form a single measure of current SES $(r=0.34, p<0.001)$. Since education and annual income are 
measured on different scales, standardization of these variables allows them to be combined into a single variable. This method of standardizing and then combining variables has been used elsewhere to create a global SES value in adult developmental research (e.g., Westerhof and Barrett, 2005) and elsewhere in psychological research in general (e.g., McKenna and Bargh, 1998).

\section{Chronic conditions}

Chronic conditions represents the presence of any of 24 chronic physical health conditions (e.g., allergies, asthma, arthritis, diabetes, heart disease, and effects of stroke) that have lasted 6 months or more and have been diagnosed by a health professional. For each participant, total number of chronic conditions was computed and this total was then re-coded to yield a measure of no chronic conditions ( 0 ), one chronic condition (1), two chronic conditions (2), three chronic conditions (3), and four or more chronic conditions (4).

\section{ANALYSES}

Three linear regression models were constructed to examine the association of childhood trauma with chronic illness in adulthood, the potential role of mental health and SES in adulthood as mediators, and the role of mental health and SES as buffers. Control variables and childhood trauma were included in the first model of each set of analyses to examine their unique contribution to chronic conditions. In the second model, mental health and SES were added simultaneously to allow a comparison of multiple potential mediators. The method used to test multiple mediators uses bootstrapping to generate a reference distribution, which is then used for confidence interval estimation and significance testing (Preacher and Hayes, 2008). This method also allows an examination of the extent to which the mediators independently contribute to an explanation of the association of the focal variable with the outcome variable as well as a comparison between mediators.

In the third model, we add the childhood trauma by mental health and childhood trauma by SES interactions. Any significant interactions were probed with an online calculator used to determine the simple slopes of the moderator from lower $(M-1 \mathrm{SD})$ to higher $(M+1 S D)$ ratings of childhood trauma (Preacher et al., 2006). Continuous variables in the regression models were meancentered to generate the required input for the online calculator used to interpret significant interactions.

\section{RESULTS}

The average age group for the sample was $5.86(\mathrm{SD}=2.70)$ which corresponded approximately to the 40- to 44-year age group. Women made up approximately 54\% of the sample. Approximately $14 \%$ of the sample consisted of visible minorities and a more detailed breakdown of race and ethnicity was not possible in the public access data. Fifty-nine percent of the participants were married, and approximately $18 \%$ had not worked during the past week. The average number of childhood traumas reported was less than $1(M=0.71, \mathrm{SD}=0.81)$. Fifty percent of the participants reported no childhood trauma, $27 \%$ reported one, and $23 \%$ reported two or more. The three most frequently mentioned childhood traumas were an event that scared the participant (20\%), family problems related to parental abuse of alcohol or drugs (18\%), and spending
2 or more weeks in the hospital (17\%). The average number of chronic conditions was greater than $1(M=1.30, \mathrm{SD}=1.33)$. Thirtyseven percent reported no chronic conditions, $26 \%$ reported having one, $17 \%$ reported $2,9 \%$ reported 3 , and $11 \%$ reported four or more. The three most frequently mentioned chronic conditions were allergies (25\%), back problems (21\%; excluding fibromyalgia or arthritis), and arthritis or rheumatism (17\%). On average, participants had high ratings of mental health $(M=3.99, \mathrm{SD}=0.92)$. Adult SES was calculated using standardized values for education and income $(M=0.09, \mathrm{SD}=0.80)$. The average level of education was "some post-secondary education," and average income was in the range of \$30,000-50,000.

Regression analyses showed that all the control variables were significantly associated with chronic illness, and the more childhood traumatic events participants reported, the more chronic conditions they had (Table 1, Model 1). The higher the ratings of mental health were, the fewer chronic conditions participants reported. Greater SES was significantly associated with having fewer chronic conditions (Table 1, Model 2). Both mental health and SES were associated with chronic conditions, and analyses were conducted to determine the degree to which the association between traumatic events and chronic conditions was accounted for by each of these potential mediators (Figure 1; Preacher and Hayes, 2008). First, the total effect $(c)$ of childhood trauma on chronic illness was significant $(B=0.30, p<0.001)$ and compared to the total effect, the direct effect $\left(c^{\prime}\right)$ of childhood trauma on chronic illness is somewhat reduced $(B=0.27, p<0.001)$ with the addition of mental health and SES to the model. The indirect effects $\left(a_{1} b_{1}\right.$ and $a_{2} b_{2}$ paths) for both mediators were statistically significant (mental health, point estimate $=0.03, \mathrm{SE}=0.004 ; z=9.11, p<0.001$; SES, point estimate $=0.003, \mathrm{SE}=0.001, z=2.67, p<0.05)$. Finally, the test of the contrast between mental health and SES was significant (point estimate $=0.03, \mathrm{SE}=0.004 ; z=7.76, p<0.001$ ), demonstrating that self-rated mental health was a significantly greater mediator than SES.

Only the trauma by SES interaction was statistically significant (Table 1, Model 3). To interpret this interaction, we compared the simple slopes for the association of childhood traumatic events with chronic illness at low levels of SES $(M-1 \mathrm{SD})$ and higher levels of SES $(M+1 S D$; Preacher et al., 2006). As shown in Figure 2, at lower levels of SES $(M-1 \mathrm{SD})$ the more childhood traumatic events participants experienced, the more chronic conditions they reported $(b=0.31, p<0.001)$. However, this pattern was weaker at higher levels of SES $(M+1 \mathrm{SD})$ such that higher ratings of childhood traumatic events were also associated with more chronic conditions, but to a lesser extent than for those at lower levels of SES $(b=0.22$, $p<0.001)$. It is also worth noting that at low levels of childhood trauma, the high and low SES lines converge, but at higher levels of childhood trauma, the high and low SES lines diverge.

\section{DISCUSSION}

The findings indicate that the greater risk of chronic health conditions in adulthood found among those who experienced childhood trauma may be the result of a process of cumulative disadvantage. Specifically, those with a history of trauma in childhood had more chronic conditions in adulthood and this was partially explained by both their lower levels of mental health as well as their diminished 
Table 1 | Unstandardized coefficients for regression models examining associations of demographics, childhood traumas, mental health, and socioeconomic status with chronic illness.

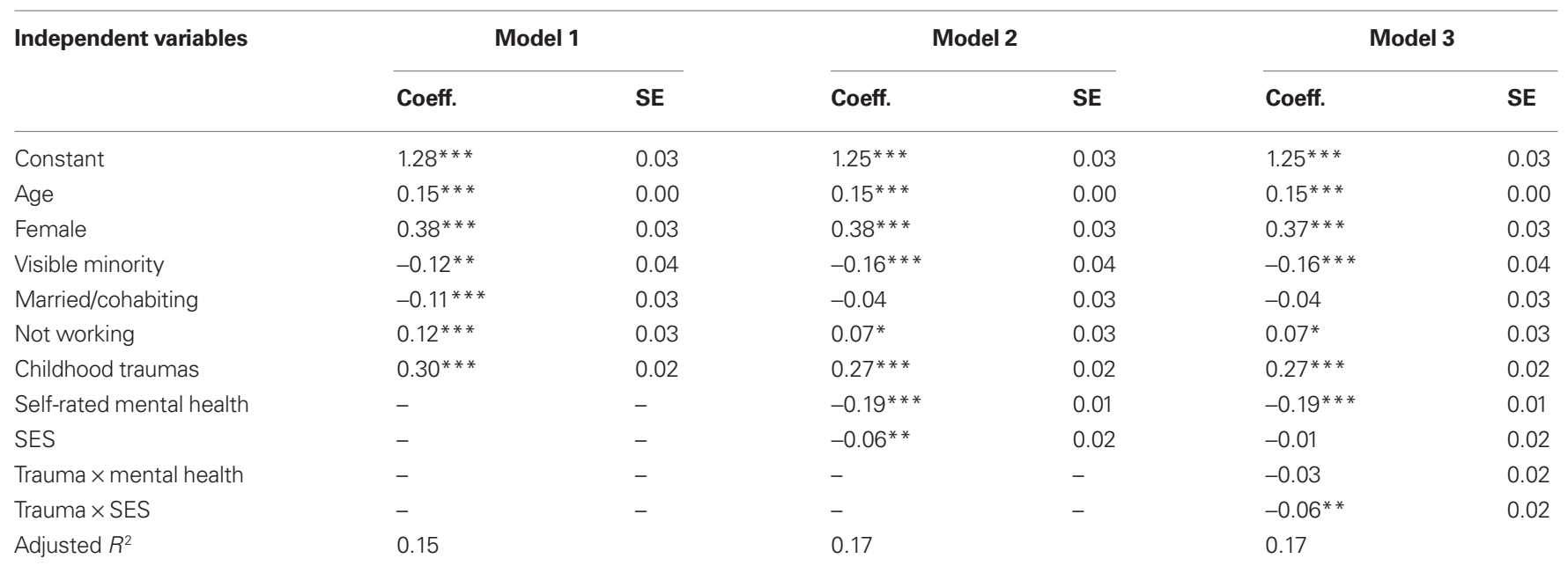

$n=9,301 ;{ }^{*} p<0.05,{ }^{*} p<0.01,{ }^{* * *} p<0.001$.

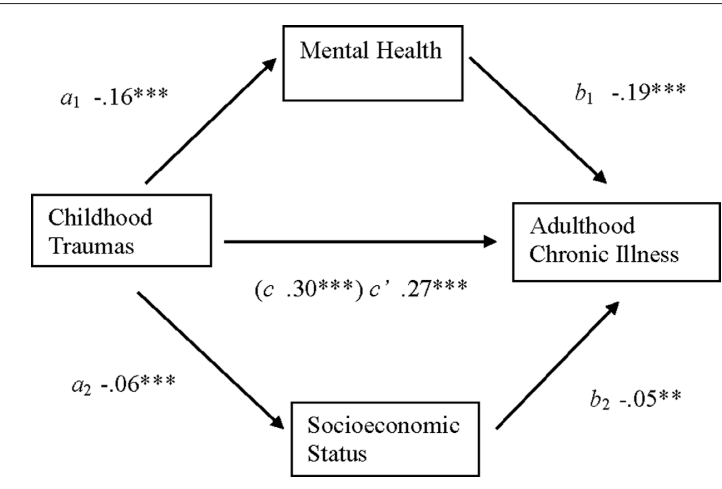

FIGURE 1 | Association between childhood trauma and chronic illness in adulthood mediated by self-rated mental health and socioeconomic status. The value in parentheses is the unstandardized regression coefficient for the association between childhood traumas and adulthood chronic illness before the addition of self-rated mental health and socioeconomic status to the model. $n=9,301 ; * * 0.01 * * *, p<0.001$.

socioeconomic resources. However, mental health contributed more to this analysis than SES did, highlighting the benefit of testing multiple mediators simultaneously.

In addition, the history of trauma by SES interaction was significant suggesting that socioeconomic resources may act as a buffer or protective factor for those with a history of trauma. It is possible that SES enables access to counseling and treatment, and more effective coping and adaptation in general. The spreading interaction shows that SES may be a particularly important resource for those with a history of trauma and is consistent with the differential susceptibility model (Belsky and Pluess, 2009) which suggests that individuals differ in their plasticity or the degree to which they are influenced by environmental forces. In the present case, those with a history of trauma may be most influenced by the benefits of higher SES and detrimental impact of lower SES relative to those who did not experience childhood traumas.
Strengths of the present study include the breadth of scope of how we measured trauma and chronic illness and the use of a population-based sample rather than clinical or convenience samples, which enhances the generalizability of findings. MacMillan et al. (1997) and Higgins and McCabe (2001) have argued that few studies explore the impacts of more than one experience of trauma. Further, many studies examine the impact of trauma on one type of chronic illness such as gastrointestinal disorders and migraines (Goodwin et al., 2003) or chronic fatigue (Heim et al., 2009). We find that an accumulation of traumatic events in childhood is associated with the number of chronic conditions participants reported supporting the idea that experiences of multiple traumas have a significant impact on health (Turner and Lloyd, 1995; Higgins and McCabe, 2001). Exploration of the explanatory and protective factors associated with this outcome provides a clearer picture of the pathways of cumulative disadvantage following trauma.

Several clinical and policy implications arise from this study. From a public health perspective, findings that suggest better mental health buffers chronic illness has implications for public health expenditures on illness and the need for preventative resources such as counseling (psychotherapy) services and income supports. Wethington et al. (2008) advocate for interventions to prevent or reduce potential psychological harm that may result from traumatic events. Literature indicates that effective treatments to reduce psychological harm following trauma include: individual and group forms of cognitive behavior therapy (Wethington et al., 2008), psychoeducation (Lubin et al., 1998; Briere and Scott, 2006), and emotional processing and narrative approaches (Briere and Scott, 2006). Although we found adult SES to be a potentially helpful buffer against chronic illnesses for people who have experienced trauma, the likelihood of experiencing trauma is itself associated with lower income and education (Hyman, 2000; MacMillan, 2000; Zielinski, 2009; Kunst et al., 2010). Consequently, addressing low SES through educational and welfare policies will have an impact at the population health level by reducing potential exposure to trauma. 


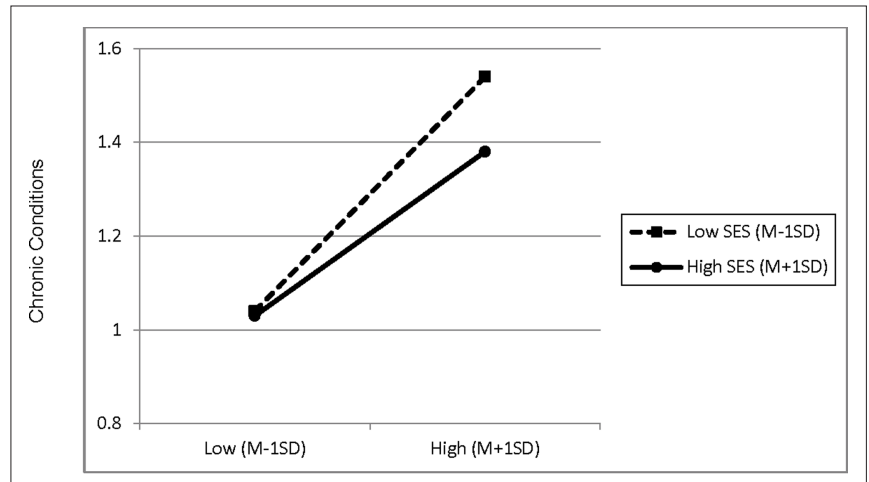

Childhood Traumas

(Mean-centred)

note: $\mathrm{SES}=$ socioeconomic status

FIGURE 2 |Association between childhood trauma and chronic illness in adulthood moderated by socioeconomic status.

Although the findings in this study contribute to an understanding of why childhood trauma may be associated with chronic conditions in adulthood and suggest a potential buffer, there are some important limitations to address. First, the data are crosssectional, precluding causal claims. Second, childhood trauma has been shown to have substantial long-term negative consequences on social functioning (Briere and Jordan, 2009; Mulvihill, 2005). Measures of social support and social functioning were not available in the module that contained measures of childhood trauma, and thus, could not be tested as potential mediators or moderators. Third, we were not able to assess the relationships between parental SES in childhood and SES in adulthood as neither parental income nor educational attainment was available in the data

\section{REFERENCES}

American Psychiatric Association. (2000). Diagnostic and Statistical Manual of Mental Disorders, 4th Edn. Washington, DC: American Psychiatric Association.

Belsky, J., and Pluess, M. (2009). Beyond diathesis stress: differential susceptibility to environmental influences. Psychol. Bull. 135, 885-908.

Breslau, N. (2009). The epidemiology of trauma, PTSD, and other posttrauma disorders. Trauma Violence Abuse 10, 198-210.

Breslau, N., Chilcoat, H. D., Kessler, R. C., and Davis, G. C. (1999). Previous exposure to trauma and PTSD effects of subsequent trauma: results from the Detroit area survey of trauma. Am. J. Psychiatry 156, 902-907.

Briere, J., and Jordan, C. E. (2009). Childhood maltreatment, intervening variables, and adult psychological difficulties in women. Trauma Violence Abuse 10, 375-388.

Briere, J., and Scott, C. (2006). Principles of Trauma Therapy: A Guide to Symptoms, Evaluation, and Treatment. Thousand Oaks, CA: Sage.
Currie, J., and Spatz Widom, C. (2010). Long-term consequences of child abuse and neglect on adult economic well-being. Child Maltreat. 15, 111-120.

Dannefer, D. (2003). Cumulative advantage/disadvantage and the life course: cross-fertilizing age and social science theory. J. Gerontol. 58B, S327-S337.

Elder, G. (1994). Time, human agency, and social change: perspectives on the life course. Soc. Psychol. Q. 57, 4-15.

Gilbert, R., Spatz Widom, C., Browne, K., Fergusson, D., Webb, E., and Jansan, S. (2009). Burden and consequences of child maltreatment in high-income countries. Lancet, 373, 68-81.

Goodwin, R. D., Hoven, C. W., Murison, R., and Hotopf, M. (2003).Association between childhood physical abuse and gastointestinal disorders and migraine in adulthood. Am. J. Public Health 93, 1065-1067.

Greenfield, E. A. (2010). Child abuse as a life-course social determinant of adult health. Maturitas 66, 51-55.

Greenfield, E. A., and Marks, N. F. (2009). Profiles of physical and psychological

set. A measure of parental SES during a participant's childhood or parental educational attainment would help to clarify whether current SES provides a buffer against negative consequences of childhood traumas, or if current SES is simply an extension of the effects of childhood SES. Fourth, the cross-sectional nature of the data also made it impossible for us to determine whether mental health and SES are best conceptualized as mediators or moderators. However, given the support that exists for both possibilities, we took the opportunity to test both in the hopes of stimulating future research on causal mechanisms.

Areas for future research include the use of longitudinal data to explore causal pathways of cumulative disadvantage. In addition, there are diverse approaches to measuring occurrence of trauma, ranging from use of police reports (Trocme et al., 2003) to broader measures of trauma that do not require direct threat of life or health (Briere and Scott, 2006). A comparison of various measures would allow an examination of their relative predictive impact on health and mediating factors following the experience of trauma. The literature also suggests the significant impact from abuse that is more intimate in nature (e.g., sexual abuse) and intentional acts of violence (Briere and Scott, 2006). Thus future research should examine outcomes of cumulative disadvantage for different types of trauma experienced in childhood. Exploration of additional social determinants of health such as social support and physical activity as potential mediators is also warranted. These are potential avenues to explore that would help give us a fuller understanding of the life course dynamics of trauma and consequences for health and well-being.

\section{ACKNOWLEDGMENTS}

The authors would like to thank Karen Gallant, Richard Eibach, and Natasha Kirkham for helpful comments at all stages of this research project.

violence in childhood as a risk factor for poorer adult health: evidence from the 1995-2005 National Survey of Midlife in the United States. J. Aging Health 21, 943-966.

Heim, C., Nater, U. M., Maloney, E., Boneva, R., Jones, J. F., and Reeves, W. C. (2009). Childhood trauma and risk for chronic fatigue syndrome. Arch. Gen. Psychiatry 66, 72-80.

Higgins, D. J., and McCabe, M. P. (2001). Multiple forms of child abuse and neglect: adult retrospective reports. Aggress. Violent Behav. 6, 547-578.

Hyman, B. (2000). The economic consequences of child sexual abuse for adult lesbian women. J. Marriage Fam. 62, 199-211.

Klein, S., and Alexander, D. A. (2009). Epidemiology and presentation of post-traumatic disorders. Psychiatry 8, 282-287.

Kunst, M. J., Bogaerts, S., Wilthagen, T., and Winkel, F. W. (2010). Income attainment among victims of violence: results from a preliminary study. Soc. Indic. Res. 95, 169-180.

Lubin, H., Loris, M., Burt, J., and Read Johnson, D. (1998). Efficacy of psy- choeducational group therapy in reducing symptoms of posttraumatic stress disorder among multiply traumatized women. Am. J. Psychiatry 155, 1172-1177.

MacMillan, R. (2000). Adolescent victimization and income deficits in adulthood: rethinking the costs of criminal violence from a life course perspective. Criminology 38 , 553-587.

MacMillan, H. L., Fleming, J. E., Trocme, N., Boyle, M. H., Wong, M., Racine, Y. A., Beardslee, W. R., and Offord, D. R. (1997). Prevalence of child physical and sexual abuse in the community. J. Am. Med.Assoc. 278, 131-135.

McKenna, K.Y.A., and Bargh, J.A. (1998). Coming out in the age of the Internet: identity "de-marginalization" through virtual group participation. J. Pers. Soc. Psychol. 75, 681-694.

Melchior, M., Moffitt, T. E., Milne, B. J., Poulton, R., and Caspi,A. (2007). Why do children from socioeconomically disadvantaged families suffer from poor health when they reach adulthood? A life-course study. Am. J. Epidemiol. 166, 966-974. 
Mulvihill, D. (2005). The health impact of childhood trauma: an interdisciplinary review, 1997-2003. Issues Compr. Pediatr. Nurs. 28, 115-136.

O'Rand,A.M., and Hamil-Luker, J. (2005). Process of cumulative adversity: childhood disadvantage and increased risk of heart attack across the life course. J. Gerontol. 60, S117-S124.

Paolucci, E. O., Genius, M., and Violato, C. (2001). A meta-analysis of the published research on the effects of child sexual abuse. J. Psychol. 135, 17-36.

Pearlin, L. I., Schieman, S., Fazio, E. M., and Meersman, S. C. (2005). Stress, health, and the life course: some conceptual perspectives. J. Health Soc. Behav. 46, 205-219.

Pine, D. S., and Cohen, J. (2002). Trauma in children and adolescents: Risk and treatment of psychiatric sequelae. Biol. Psychiatry 51, 519-531.

Preacher, K. J., Curran, P. J., and Bauer, D. J. (2006). Computational tools for probing interaction effects in multiple linear regression, multilevel modeling, and latent curve analysis. J. Educ. Behav. Stat. 31, 437-448.
Preacher, K. J., and Hayes, A. F. (2008). Asymptotic and resampling strategies for assessing and comparing indirect effects in multiple mediator models. Behav. Res. Methods 40, 879-891.

Springer, K. W., Sheridan, J., Kuo, D., and Carnes, M. (2003). The long-term health outcomes of childhood abuse: an overview and call to action. J. Gen. Intern. Med. 18, 864-870.

Statistics Canada. (2006a). Canadian Community Health Survey (CCHS) Cycle 3.1 (2005): Public Use Micro Data File (PUMF) User Guide. Ottawa: Statistics Canada.

Statistics Canada. (2006b). Canadian Community Health Survey (CCHS) Cycle 3.1 (2005): Public Use Micro Data File (PUMF) Integrated Derived Variable (DV) and Grouped Variable Specifications. Ottawa: Statistics Canada.

Stein, M. B., Walker, J. R., Hazen, A. L., and Forde, D. R. (1997). Full and partial posttraumatic stress disorder: findings from a community survey. Am. J. Psychiatry 154, 1114-1119.

Taylor, S. E. (2010). Mechanisms linking early life stress to adult health outcomes. Proc. Natl.Acad. Sci. U.S.A. 107, 8507-8512.
Trocme, N. M., Tourigny, M., MacLaurin, B., and Fallon, B. (2003). Major findings from the Canadian incidence study of reported child abuse and neglect. Child Abuse Negl. 27, 1427-1439.

Turner, R. J., and Lloyd, D. A. (1995). Lifetime traumas and mental health: the significance of cumulative adversity. J. Health Soc. Behav. 36, 360-376.

Westerhof, G. J., and Barrett, A. E. (2005). Age identity and subjective well-being: a comparison of the United States and Germany. J. Gerontol. Soc. Sci. 60B, 129-136.

Wethington, H. R., Hahn, R. A., FuquaWhitley, D. S., Sipe, T. A., Crosby, A E., Johnson, R. L., Liberman, A. M., Mościcki, E., Price, L. N., Tuma, F. K., Kalra, G., and Chattopadhyay, S. K. (2008). The effectiveness of interventions to reduce psychological harm from traumatic events among children and adolescents: a systematic review. Am. J. Prev. Med. 35, 287-313.

Wickrama, K. A. S., Conger, R. D., and Abraham, W. (2005). Early risks and later health: the intergenerational transmission of socioeconomic adversity through mental disorder and physical illness. J. Gerontol. 60, S125-S129.

Zielinski, D.S. (2009). Child maltreatment and adult socioeconomic well-being. Child Abuse Negl. 33, 666-678.

Conflict of InterestStatement: Theauthors declare that this research was conducted in the absence of any commercial or financial relationships that could be construed as a potential conflict of interest.

Received:03July2010; accepted:27 December 2010; published online: 31 January 2011.

Citation: Mock SE and Arai SM (2011)

Childhood trauma and chronic illness in adulthood: mental health and socioeconomic status as explanatory factors and buffers. Front. Psychology 1:246. doi: 10.3389/fpsyg.2010.00246

This article was submitted to Frontiers in Developmental Psychology, a specialty of Frontiers in Psychology.

Copyright (C) 2011 Mock and Arai. This is an open-access article subject to an exclusive license agreement between the authors and Frontiers Media SA, which permits unrestricted use, distribution, and reproduction in any medium, provided the original authors and source are credited. 